NUCLEAR INSTRUMENTS AND METHODS 135 (1976) 59-529; (C) NORTH-HOLLAND PUBLISHING Co.

\title{
A METHOD TO IDENTIFY NUCLEAR REACTIONS REGISTERED IN IONOGRAPHIC DETECTORS
}

\author{
M. ORTEGA, A. VIDAL-QUADRAS, M. TOMÁS, V. GANDFA. \\ Departamento de Física Fundanental, Universidad Autónoma de Barcelona, Spain \\ and \\ C. JACQUOT \\ Service d'Application des Détecteurs Visuets, Centre de Recherches Nucleaires, Strasbourg, France
}

Received 15 Jantary 1976

\begin{abstract}
A method is established to examine all compatible assignations of charge and mass number to the target and emergent prongs of an event registered in an ionographic detector (such as nuclear emulsion or silver chloride crystals) under the requirements of conservation of momentum and total energy. The kinematical parameters corresponding to the most likely configurations are determixed by a least-squares process. For the accepted hypothesis, the probability associated to the minimized value of the chi-square function and to the number of degrees of freedom is computed. The correctness of the method is tested by means of simulated events.
\end{abstract}

\section{Introduction}

The study of low energy (below meson production) nuclear reactions by means of ionographic detectors that register the whole interaction over the space $4 \pi \mathrm{sr}$ has proved valuable in the last years mainly in connection with:

a) determination of reaction and production cross sections of astrophysical interest ${ }^{1}$ ),

b) study of reaction mechanisms and spectroscopy of light nuclei ${ }^{2,3}$ ).

The method followed in all these works ${ }^{4}$ ) to identify the target and emergent fragments has been the examination of all compatible assignations of charge and mass number to the different prongs of the interaction under the requirements of conservation of energy and momentum. Although complementary ionization measures were performed occasionally to distinguish between charge one and two, no other decision criterion was used to fix the identity of the target and emergent fragments that the comparison between the values of conservation of momentum and energy of the different hypotheses. In the frequent case of a "doubtful" event with several configurations with similar conservation values a decision was not possible and the event had to be rejected. In other cases, a configuration was chosen with a considerable degree of subjectivity in the election. The consequences of this way of identifying the measured events were on one hand a decrease in the statistics because of rejected events, and on the other, the introduction of uncontrollable errors in the physical parameters to be determined.
The aim of the present work is to establish an iden tification method free of subjectivity and statistically rigorous, permitting to reject the non-physical hypotheses and assign to the possible ones a normalized probability.

The problem is not new and was faced a long time ago by the bubble chamber ${ }^{5,6}$ ). We have followed very closely the bubble chamber method and adapted it to our case. There are two strong differences, however, between both situations:

1) the geometry is much simpler for events registered in ionographic detectors (no track curvature);

2) the number of hypotheses to be examined is much greater. Because of this last fact, the adaptation of an identification method originally conceived by elementary particle physicists to decide among a few configurations for one event to typical intermediate energy nuclear processes, where for a fixed beam, energy and target, hundreds or even thousands of possibilities have to be considered, would not have been profitable without the existence of the very big and fast computers developed in the last years.

\section{General description of the method}

The method has two well-cut parts:

1) determination of geometrical parameters,

2) kinematical analysis.

The first part converts the geometrical quantities directly measured on the event and their errors into range and polar angles $R \pm \Delta R, \theta \pm \Delta \theta, K \pm \Delta K$ and their errors for each prong.

The second part can be divided into several sections: 
a) computation of momentum, kinetic energy and

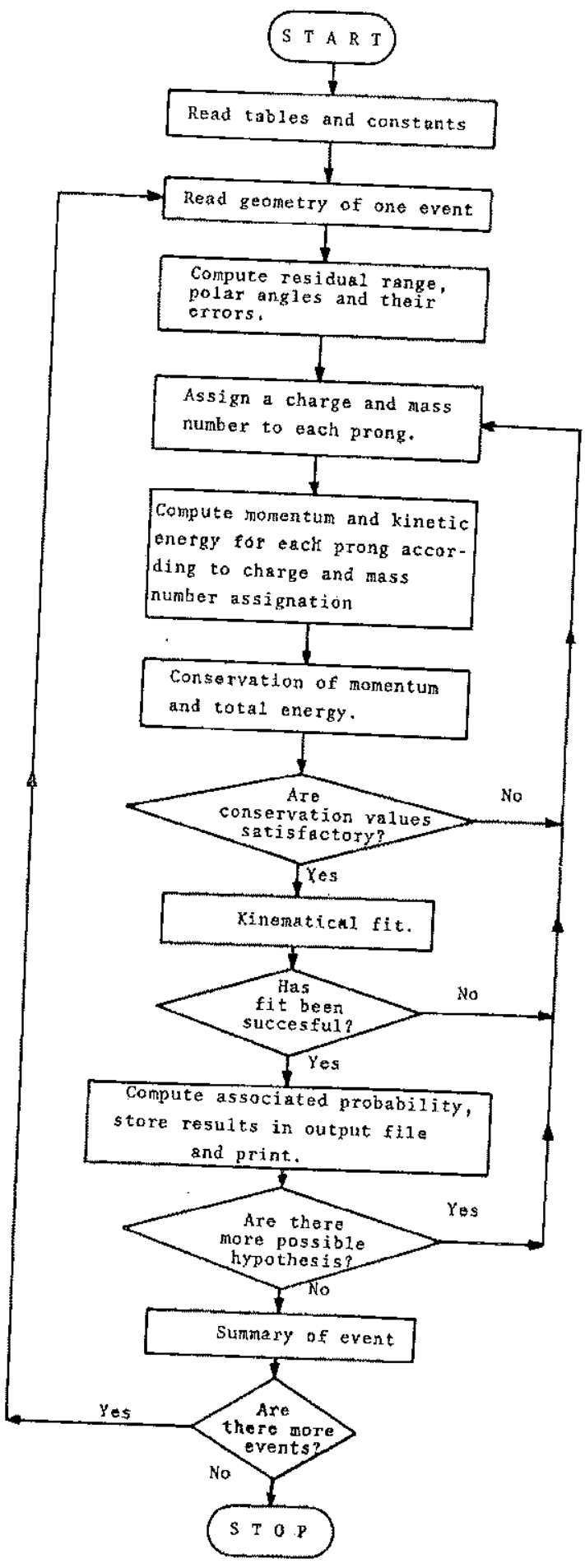

Fig. 1. Flux diagram of identification process. their errors for each prong by means of the measured value of residual range and its error and the rangeenergy relation;

b) generation of all compatible hypotheses of charge and mass number for each prong;

c) rejection of non-physical hypotheses under the requirements of momentum and energy conservation taking into account experimental errots;

d) determination of the values of momentum and polar angles for each prong that minimize the chisquare function and satisfy simultaneously the conservation equations;

e) computation for each fitted hypothesis of the probability associated to the minimized chi-square and to the number of degrees of freedom. Decision on acceptability according to probability value.

In the next sections we give more details on the sections of the second part. In fig. 1 we present a flux diagram ilustrating the method. We do not give further explanation in this paper about the measure of the geometry of one event and transformation of geometrical measures into $R \pm \Delta R, \theta \pm \Delta \theta, K \pm \Delta K$, as this is a well known topic for researchers using nuclear emulsion or any other visual detector. However, we want to emphasize, obvious as it is, that a careful measure of the geometry and a correct determination of experimental errors is basic for the ulterior good evolution of the kinematical fit

\section{Range-energy relation}

The precise knowledge of the range-energy relation in the detector for the different jons is most necessary to establish the kinematics of the registered reactions. As is well known ${ }^{7}$ ) the residual range of an ion of mass $M$ in proton mass units, charge number $Z$ and velocity $\beta c$ is given by:

$R(\beta)=\left(M / Z^{2}\right)\left[\lambda(\beta)+B_{\bar{z}}(\beta)\right]$,

were $\lambda(\beta)$ stands for the residual range in the detector of an ideal proton of velocity $\beta c$ that does not suffer close collisions with nuclei nor experiences electron capture, and $B_{z}(\beta)$ is the range extension function. For the computation of $\lambda(\beta)$ and $B_{z}(\beta)$ we have followed the method established by Benton and Henke ${ }^{8}$ ). The range-energy table for the proton and the values of $\langle A / Z\rangle$ and mean ionization potential in the particular detector are given as input data and must be previously calculated ${ }^{9}$ ).

However, the experimentally measured parameter is the residual range and the kinetic energy must be calculated herefrom. For this purpose, the inverse func- 
TABLE 1

Inverse function of eq. (1) for ${ }^{7} \mathrm{Be}$.

\begin{tabular}{ccc}
$\begin{array}{c}E \\
(\mathrm{MeV})\end{array}$ & $\begin{array}{c}R \\
(\mu \mathrm{m})\end{array}$ & $\begin{array}{c}E^{*} \\
(\mathrm{MeV})\end{array}$ \\
\hline 0.0625 & 0.24 & 0.0629 \\
0.1250 & 0.40 & 0.1253 \\
0.2500 & 0.66 & 0.2503 \\
0.5000 & 1.12 & 0.5007 \\
1.0000 & 1.93 & 1.0008 \\
2.0000 & 3.36 & 2.0002 \\
4.0000 & 5.89 & 4.0016 \\
8.0000 & 11.06 & 8.0007 \\
16.0000 & 25.84 & 15.9983 \\
32.0000 & 70.79 & 31.9989 \\
64.0000 & 216.14 & 64.0050 \\
128.0000 & 704.58 & 127.9985 \\
\hline
\end{tabular}

tion of eq. (1) has been constructed, i.e., a function that gives the kinetic energy corresponding to a set of values of mass, charge and residual range. In this function a range-energy table is built locally about an approximate value of the range computed with the range of proton of the same $\beta$. The value of the kinetic encrgy is then interpolated in this table. The reproductibility of the value of the kinetic energy by means of this procedure is illustrated in table 1 . The first column shows several values of the kinetic energy ranging from zero up to $130 \mathrm{MeV}$, the second column corresponding values of range calculated with eq. (1) and the third column the values of kinetic energy computed with the range values of the second column.

\section{Momentum assignation}

All possible values of momentum and energy associated with all possible identities for each prong are computed with the corresponding measured value of residual range. For the incoming beam, whose identity is known, the problem is reduced to compute its energy with the incorporated proton range-energy table. For emergent particles, whose identities are not known, all permitted stable muclides must be considered. Charge and mass number conservation forbid certain configurations that are eliminated previously to kinematical fit in the following way: The maximum allowed values of charge and mass number $Z_{m}, A_{\mathrm{m}}$ for each prong of a star with $N$ visible prongs are

$Z_{\mathrm{m}}=Z_{\mathrm{i}}+Z_{\mathrm{t}}-(N-2)$,

$A_{\mathrm{m}}=A_{\mathrm{i}}+A_{\mathrm{t}}-(N-2)$,

where $i$ and $t$ stand for incident beam and target respectively.
If $k$ is the order number of the $\left(Z_{\mathrm{m}}, A_{\mathrm{m}}\right)$ nuclide in the incorporated table of stable nuclides, kinetic energy $T_{i j}$ and momentum $p_{i j}$ are calculated corresponding to residual range $R_{i}$ of prong $i, i=1,2, \ldots, N$ for stable nuclides $j, j=1,2, \ldots, k$. The error in the kinematic energy is estimated computing the values $T_{i j}-\Delta_{1} T_{i j}, T_{i j}+\Delta_{2} T_{i j}$ corresponding to $R_{i}-\Delta R_{i}$, $R_{i}+\Delta R_{i}$

$T_{i j}-\Delta_{1} T_{i j}<T_{i j}<T_{i j}+\Delta_{2} T_{i j}$,

where $\Delta_{1} T_{i j} \neq \Delta_{2} T_{i l}$ because the relation between range and energy is not linear. The greatest of $\Delta_{1} T_{i j}$ and $A_{2} T_{i j}$ is taken as error in the kinetic energy.

Other sources of error in the determination of the kinetic energy that must be taken into account are the intrinsic error in the proton range-energy relation and the accuracy of the expression used for $B_{z}(\beta)$.

If the kinetic energy of assignation $j$ is greater than the incident kinetic energy all hypotheses corresponding to nuclides with order number greater than $j$, i.e., $j+1, \ldots, k$, can be rejected because of the endotermic character of the nuclear reactions under study.

\section{Generation of hypotheses on charge and mass number}

Once momentum assignation has been performed, every possible hypothesis on the identity of the target and emergent fragments must be tried. These hypotheses can be previously stored in an auxiliary file (tape or fastrand). In this case the identification of each event will only require to read the stored hypotheses with a considerable saving of computer time. If the hypotheses are not already stored, they are generated and stored for later use.

To generate all hypotheses on charge and mass number for an $N$-prong interaction. unrestricted variations of the first $k$ stable nuclides must be generated. The number of variations is very large, but many among them are not compatible with mass and charge conservation. To impose this conservation, every variation on every possible target is tested. The compatible hypotheses are codified in octal system and stored in 36 bit words in order to reduce the occupation of central memory.

In table 2 we give a summary corresponding to hypothesis generation for $\mathrm{p}+\mathrm{C}, \mathrm{N}, \mathrm{O}$. We want to emphasize the importance of having the table stored in a auxiliary file in order to reduce computer time. This is clearly seen comparing the last two rows of table 2 .

\section{ISinenatical fit}

Once a hypothesis has been made on the identity 
TABLE 2

Hypothesis generation for interactions $\mathrm{p}+\mathrm{C}, \mathrm{N}, \mathrm{O}$. The number of possible emergent fragments is 37 , i.e., the stable isotopes up to ${ }^{16} \mathrm{O}$.

\begin{tabular}{lrrrr}
\hline Number of prongs & \multicolumn{1}{c}{4} & \multicolumn{1}{c}{5} & \multicolumn{1}{c}{6} \\
\hline $\begin{array}{l}\text { Number of variations } \\
\text { Number of hypotheses }\end{array}$ & 28991 & 390625 & 3200000 \\
$\begin{array}{l}\text { Computer time for hypoth- } \\
\text { esis generation }\end{array}$ & $15 \mathrm{~s}$ & $2 \mathrm{~min}$ & $10 \mathrm{~min}$ \\
$\begin{array}{c}\text { Computer time for identifi- } \\
\text { cation of one event }\end{array}$ & $4 \mathrm{~s}$ & $18 \mathrm{~s}$ & $80 \mathrm{~s}$ \\
\hline
\end{tabular}

of each emergent particle, three parameters can be associated to each prong, namely its momentum and polar angles $p_{i}, \theta_{i}, K_{i}$, in an arbitrary frame of reference. The masses corresponding to a particular hypothesis are not considered as parameters to be fitted but as constants. Every fragment is supposed to be emitted in the ground state. For an $N$-prong interaction, there are $3 N$ parameters to be estimated: $p_{i}, \theta_{i}, K_{j}, i=1, \ldots, N$. If their measured values and corresponding experimental errors are $p_{i}^{\mathrm{m}} \pm \sigma_{\rho_{i}}^{\mathrm{m}}, \theta_{i}^{\mathrm{m}} \pm \sigma_{\theta_{i}}^{\mathrm{m}}, K_{i}^{\mathrm{m}} \pm \sigma_{K_{i}}^{\mathrm{m}}$, the chirsquare function $\chi^{2}\left(p_{i}, 0_{i}, K_{i}\right)$ is defined:

$$
\begin{aligned}
\chi^{2}= & \sum_{i=1}^{N}\left\{\left(p_{i}-p_{i}^{\mathrm{m}}\right)^{2} /\left(\sigma_{p_{i}}^{\mathrm{m}}\right)^{2}+\right. \\
& \left.+\left(\theta_{i}-0_{i}^{\mathrm{m}}\right)^{2} /\left(\sigma_{\theta_{i}}^{\mathrm{m}}\right)^{2}+\left(K_{i}-K_{i}^{\mathrm{m}}\right)^{2} /\left(\sigma_{K_{i}}^{\mathrm{m}}\right)^{2}\right\}
\end{aligned}
$$

The values of the kinematical parameters $p_{i}, \theta_{i}, K_{i}$ that minimize the function $\chi^{2}\left(p_{i}, \theta_{i}, K_{i}\right)$ and verify simultaneously the four constraints given by conservation of momentum and energy must be calculated. These four contraints are:

$$
\begin{aligned}
& \sum_{i=1}^{N} p_{i} \cos \theta_{i} \cos K_{i}=0 \\
& \sum_{i=1}^{N} p_{i} \cos \theta_{i} \sin K_{i}=0 \\
& \sum_{i=1}^{N} p_{i} \sin \theta_{i}=0 \\
& E_{1}+M_{1}-\sum_{i=2}^{N} E_{l}=0
\end{aligned}
$$

where

$E_{1}:$ total energy of incident particle,

$M_{\mathrm{t}}$ : target raass,

$E_{i}$ : total energy of particle $i$.

The solution of this problem is found ${ }^{10}$ ) by the method of Lagrange multipliers. As the contraints (2) are not linear, they are developped up to first order in a neighbourhood of an approximate solution. The resulting system of $3 N+4$ equations with $3 N+4$ unknowns $\left(p_{i}, \theta_{i}, K_{i}, i=1, \ldots, N\right.$ and the four arbitrary Lagrange multipliers) is then solved. The outlined process is one step of an iterative procedure in which the measured values are taken as the initial approximate solution. The iterations are stopped or abandoned when certain conditions to be described are fulfilled.

When one hypothesis has been succesfully fitted, the probability $P\left(\chi_{0}^{2}, v\right)$ associated to the number of degrees of freedom $v$ and to the chi-square minimized value $\chi_{0}^{2}$, is computed.

A neutral particle is not visible in the detector. Hypotheses admitting one neutron are examined, the neutron being assigned in each iteration a momentum $p_{n}$ of components:

$p_{n x}=-\sum_{i=1}^{N} p_{i} \cos \theta_{i} \cos K_{i}$,

$p_{\mathrm{n} y}=-\sum_{i=1}^{N} p_{i} \cos \theta_{i} \sin K_{i}$,

$p_{\mathrm{nz}}=-\sum_{i=1}^{N} p_{i} \sin \theta_{i}$

where $N$ refers now to the number of visible prongs. The three equations concerning conservation of momentum are automatically satisfied and there is only one constraint remaining valid: the equation corresponding to the conseryation of energy. The momentum and polar angles of the neutron are non-measured variables which are calculated according to eqs. (3). In this case, the number of degrees of freedom is $v=1$.

If a particle reaches the surface of the detector before coming to rest, its direction is known, but its momentum is not. In this case, the particle is assigned in each step of the iterative process the modulus of momentum $p_{1}$ that verifies exactly the conservation of energy:

$p_{i}=\left(E_{l}^{2}-M_{i}^{2}\right)^{\frac{1}{2}}$,

where $M_{l}$ is the rest mass of the lost particle in the hypothesis under consideration and $E_{l}=M_{1}+\sum E_{i}-E_{1}$, the sum not including the incident particle nor the lost one. The momentum $p_{l}$ is a non-measured variable and there are three valid remaining constraints, those given by the conservation of momentum. The number of degrees of freedom $v=3$. 


\section{Control of iteration procedure}

\subsection{SELECTION OF HYPOTHESIS BEFORE FIT}

Submitting a given hypothesis to the kinematical fit can be unnecessary for three reasons: (a) because measured values satisfy almost exactly conservation equations; (b) because measured values are so far of satisfying conservation constraints that the fit procedure could never converge; (c) because the residual range of one prong is not compatible with the $(Z, A)$ assignation and incident energy.

Case (a) is tested comparing the values of the first member of eqs. (2) with certain minimum values. Case (b) is tested comparing the values of the first member of eqs. (2) with the sum of absolute values of experimental errors multiplied by a factor. This factor and the minimum values previously mentioned are fixed according to particular experimental conditions and given as input data.

\subsection{CONTROL OF CONVERGENCE}

An hypothesis satisfying the requirements of section 7.1 enter into the fit iteratjve procedure. Its convergence is controlled in the following way: Let $x_{i}$ be the values of kinematical parameters after iteration $i$ has been performed. The values $x_{l}$ are substituded by $\frac{1}{2}\left(x_{i-1}+x_{i}\right)$ before step $i+1$ in the following cases:

a) If the sum of modulus of differences between parameter values in consecutive steps $\sum_{j}\left|x_{i j}-x_{i-1, j}\right|$ is greater than the preceding sum

$\sum_{j}\left|x_{i-1, j}-x_{i-2, j}\right|$.

b) If the sum of absolute values of the first members of eqs. (2) after step $i$ is greater than the preceding sum.

c) If the sign of modulus of momentum of one fragment is negative after step $i$. This situation can occur with very short tracks.

d) If the sign of the $\chi_{0}^{2}$ value is negative after step $i$.

The maximum number of consecutive cut-steps of this sort that can be performed is fixed according to experimental conditions. The total allowed number of cut-steps during the fit of a particular hypothesis is also limited. When these limit values are reached, the hypothesis is considered divergent and is rejected.

\section{Assignation of probability and acceptability of fitted hypothesis}

The probability associated to the minimized value of the function and to the number of degrees of free- dom:

$P\left(\chi^{2}>\chi_{0}^{2}\right)=\int_{\chi_{0}^{2}}^{\infty} f\left(\chi^{2}\right) \mathrm{d} \chi^{2}$,

where $f\left(\chi^{2}\right)$ is the chi-square distribution function, is calculated by a recursion method $\left.{ }^{11}\right)$.

If $1 \%<P\left(\chi_{0}^{2}, v\right)<5 \%$, the hypathesis is considered doubtful and if $P\left(\chi_{0}^{2}, v\right)<1 \%$, the hypothesis is rejected. These limit values are the ones accepted in decision theory for a variable distributed according to a normal law.

\section{Examples}

We give now two examples of identified events.

The first (table 3) corresponds to a five-prong interaction which has been identified as unique solution $\alpha+{ }^{12} \mathrm{C} \rightarrow 4 \alpha$. The only accepted hypothesis has a probability $P\left(\chi_{0}^{2}, v\right)=85 \%$, but as it is unique, its normalized probability is $100 \%$,

The second example (table 4) corresponds to the identification of a six-prong interaction. There are two accepted solutions: $\alpha+{ }^{16} \mathrm{O} \rightarrow 5 \alpha$ and $\alpha+{ }^{14} \mathrm{~N} \rightarrow \mathrm{d}+4 \alpha$.

It is apparent in both examples that complete kinematics is computed for each accepted hypothesis, before and after fit, as momentum, polar angles and their errors for each prong. The minimized chi-square $\chi_{0}^{2}$ and associated probability $P\left(\chi_{0}^{2}, v\right)$ are also given. Conservation values eqs. (2) of the three components of momentum and of the total energy, before and after fit, are also computed. In the examples shown each event is headed by its geometry $R \pm \Delta R, \theta \pm \Delta \theta$, $K \pm \Delta K$ for each prong.

\section{Tests on the method}

\subsection{SimuLATION OF EVENTS}

In order to test the correctness of the method and fix the optimal values of the parameters governing previous selection, acceptability and convergence of the different hypotheses, we have worked on simulated events generated by the CERN program FOWL. As is well known, this program generates simulated events satisfying conservation of momentum and energy. Their kinematical parameters can be transformed into geometrical yariables corresponding to the particular detector and measuring process. These geometrical variables are subsequently deformed to give simulated measured values according with experimental errors supposing a random normal behaviour of the measuring process. 
8

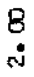

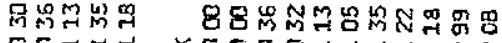

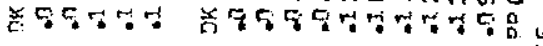

8

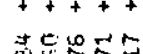

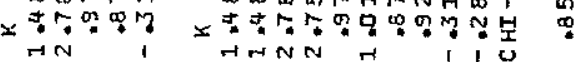

$+7+7+7++7+0$

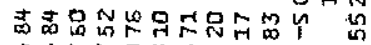

ro

w.

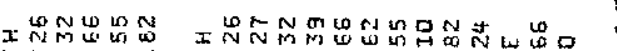

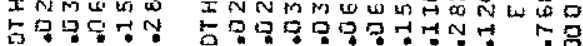
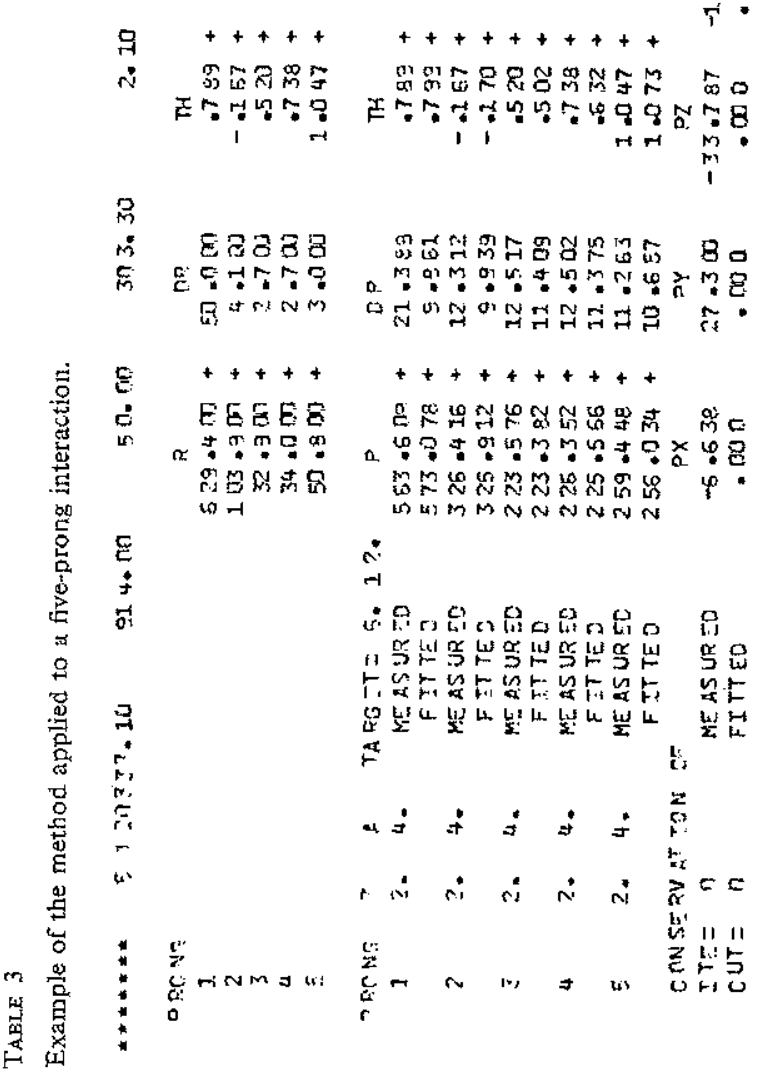

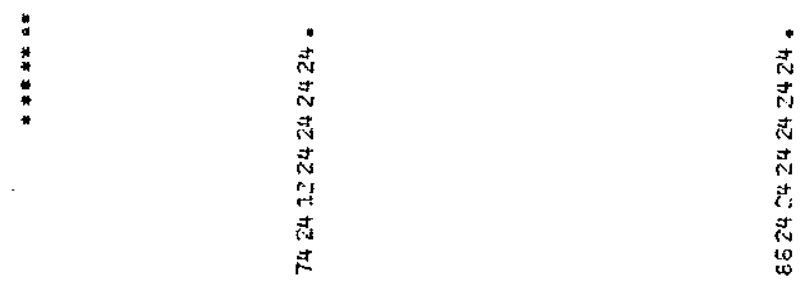

$\stackrel{8}{9}$

11
4
0
0

8

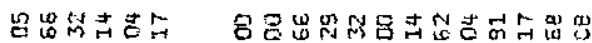

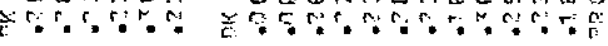

8

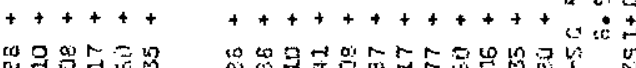

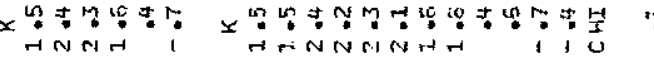

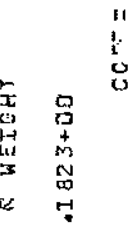

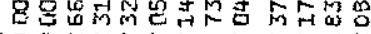

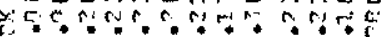

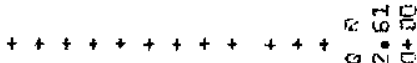

o

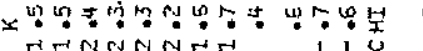

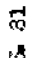

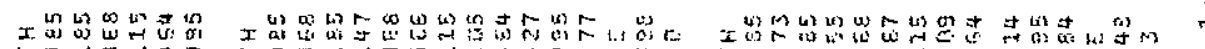

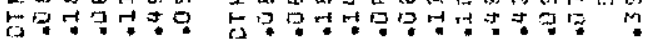

只

$++t++$

$++++++++++t$

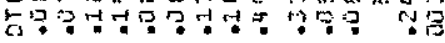

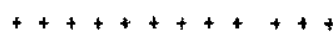

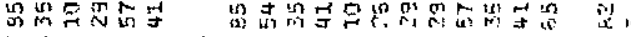

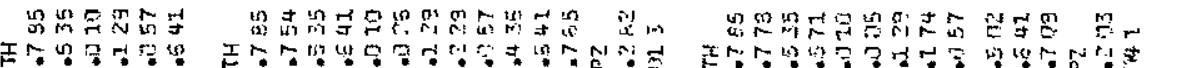
a.

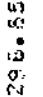

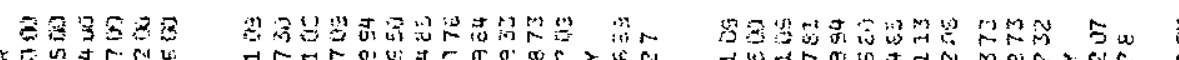

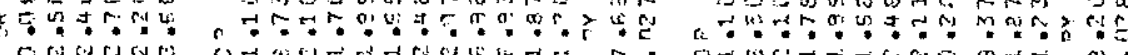

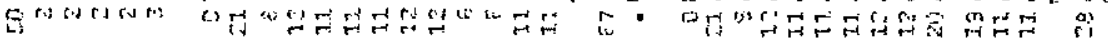

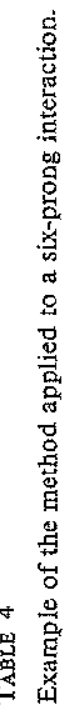

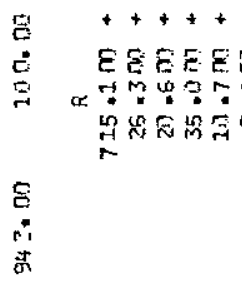

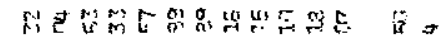

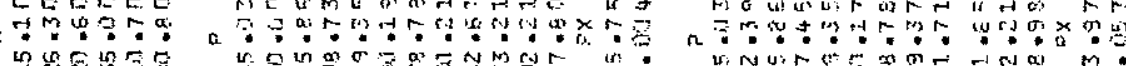

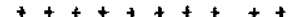

FE

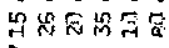

$$
\stackrel{+9}{4}
$$

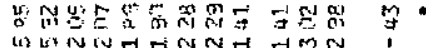
$\therefore$

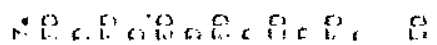

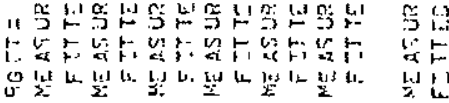

aits

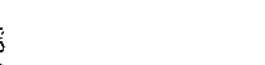

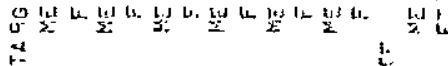

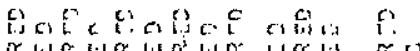
1) tid $i^{2}$ $+\therefore \therefore \therefore \therefore \therefore$

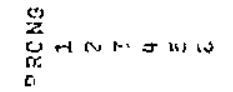
焉

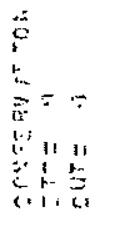
$\dot{i} \div \therefore \dot{0} ;$ 然 


\subsection{VALUES OF CONTROL PARAMETERS}

Over 2500 events were simulated corresponding to different kinematical configurations in order to cover a broad spectrum reflecting the real experiment, 2365 have been succesfully fitted with associated probability greater than $2 \%$. The rest have been rejected mainly because of difficulties of convergence. The average value of iterations necessary to fit the correct hypothesis (which has been imposed to save computer time) was 2.2. The distribution is shown in fig. 2 . The distribution of the number of cut-steps performed is

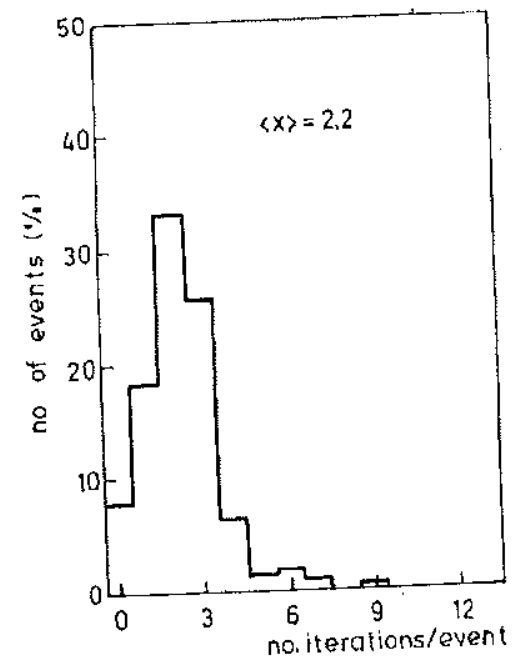

Fig. 2. Distribution of the number of iterations performed in the identification of one event over 2500 simulated events.

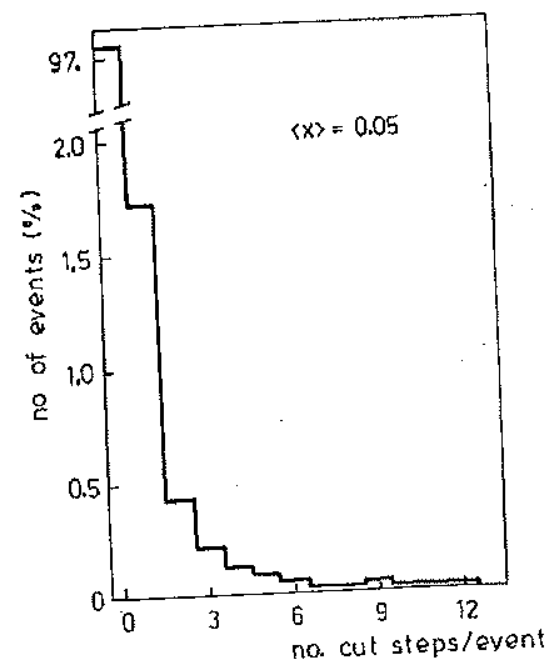

Fig. 3. Distribution of the number of cut-steps performed in the identification of one event over 2500 simulated events. shown in fig. 3 . In a $98 \%$ of cases, the correct hypothesis is fitted without a cut-step being necessary. The same tests have been done fixing an hypothesis whose configuration is close to the correct one and giving therefore quite good initial conservation values (i.e. $\mathrm{p}+{ }^{16} \mathrm{O} \rightarrow \mathrm{d}+{ }^{3} \mathrm{He}+2 \alpha$ instead of $\left.\mathrm{p}+{ }^{16} \mathrm{O} \rightarrow \mathrm{p}+4 \alpha\right)$. In these cases, the number of iterations and cut-steps necessary to fit the hypothesis are much greater. This fact could be used to select the correct hypothesis fixing sufficiently low values of control parameters, but this would imply in certain cases rejecting prematurely the correct solution, so we have chosen compromise values of a maximum of a total of 10 iterations and 10 cut-steps during the fit of one hypothesis and 3 consecutive cut-steps.

Similar considerations have led us to reject hypotheses whose initial conservation values (2) are greater than the double of the sum of absolute values of exper imental errors, and to stop the iteration procedure when conservation values (2) are smaller than 0.25

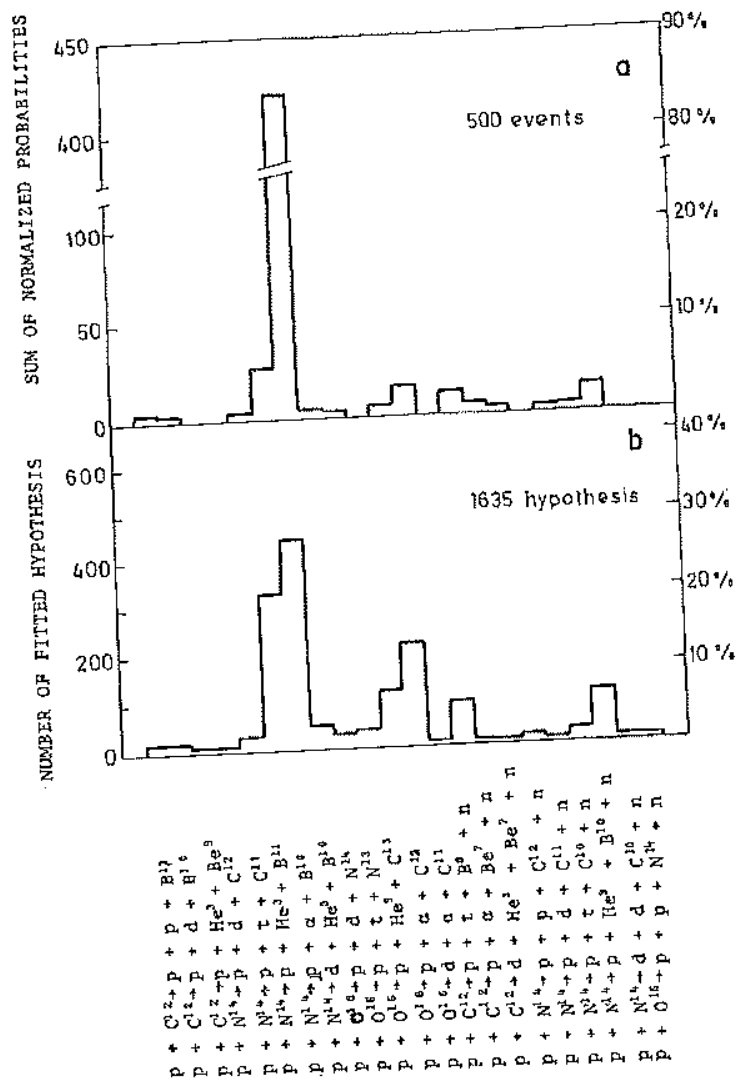

Fig. 4. Contamination of the correct solution $\mathrm{p}+14 \mathrm{~N} \rightarrow \mathrm{p}+\alpha+{ }^{10} \mathrm{~B}$ in the identification of 500 simulated events. 
$\mathrm{MeV} / \mathrm{c}$ for the three components of momentum and $0.25 \mathrm{MeV}$ for total energy.

\subsection{CONTAMINATION OF CORRECT SOLUTION}

The associated probability $P\left(\chi_{0}^{2}, v\right)$ corresponding to the correct hypothesis will usually predominate over the rest, but it can happen that incorrect hypotheses be accepted with probabilities greater than $5 \%$. As in the identification of real events the correct hypothesis is not known, it is of interest to test the degree of contamination of the good solution. In fig. 4 we show the result of the identification of 500 simulated events $\mathrm{p}+{ }^{14} \mathrm{~N} \rightarrow \mathrm{p}+\alpha+{ }^{10} \mathrm{~B}$. Histogram b represents the number of times a given hypothesis appears as solution of the event with an associated probability greater than $2 \%$. It is clear that the channel $\mathrm{p}+{ }^{14} \mathrm{~N} \rightarrow \mathrm{p}+{ }^{3} \mathrm{He}+{ }^{11} \mathrm{~B}$ appears as possible solution a number of times comparable to the correct solution. Histogram $a$ shows the sum of normalized probabilities for each hypothesis. Contamination represents the $15.5 \%$ of the accepted solutions for the 500 simulated events and the rest, $84.5 \%$ correspond to the correct solution.

\subsection{Statistical Behaviour of Kinematical Fit}

In order to study the statistical behaviour of kinematical fit and the influence of a correct estimation of geometrical errors, 2500 simulated events have been identified imposing the correct hypothesis. The $\chi^{2}$ and

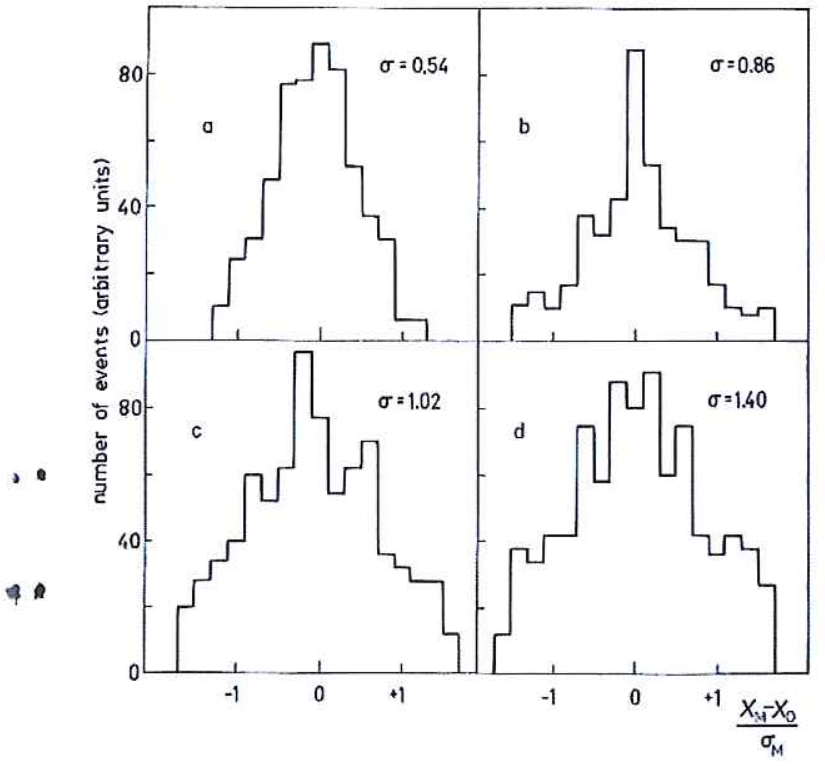

Fig. 5. Distributions of deformations of geometrical parameters in 500 simulated events $\alpha+{ }^{12} \mathrm{C} \rightarrow \alpha+{ }^{6} \mathrm{Li}+{ }^{6} \mathrm{Li}$. associated probability distributions have been examined for different deformations of the values of geometrical variables corresponding to the physically correct event (verifying exactly conservation of momentum and energy). We will call:

$x_{\mathrm{m}}$ : measured (deformed) values of kinematical parameters,

$x_{\mathrm{f}}$ : fitted values of kinematical parameters,

$x_{0}$ : values of kinematical parameters corresponding

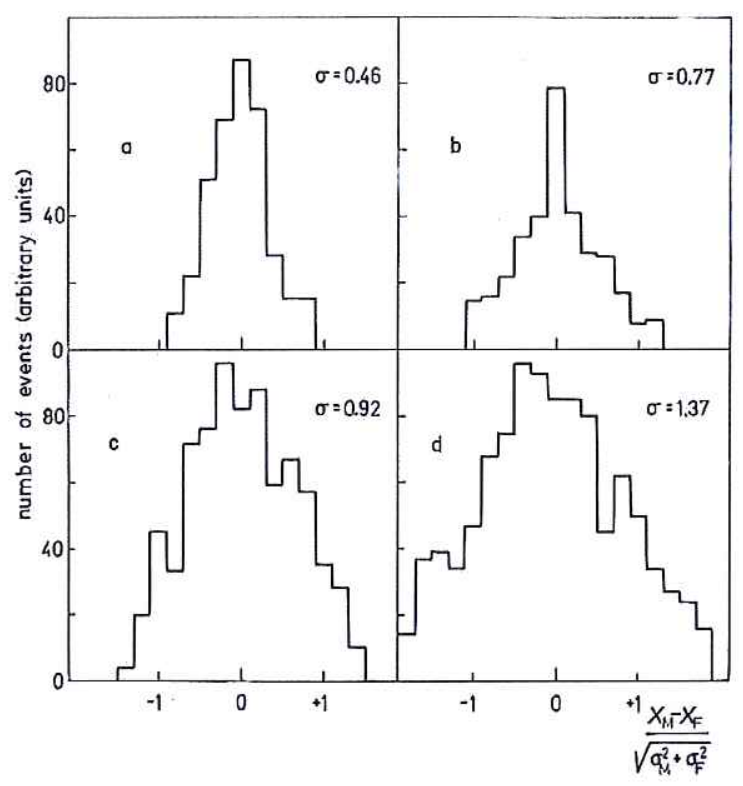

Fig. 6. Distributions of "pulls" corresponding to geometrical parameters in 500 simulated events $\alpha+{ }^{12} \mathrm{C} \rightarrow \alpha+{ }^{6} \mathrm{Li}+{ }^{6} \mathrm{Li}$.

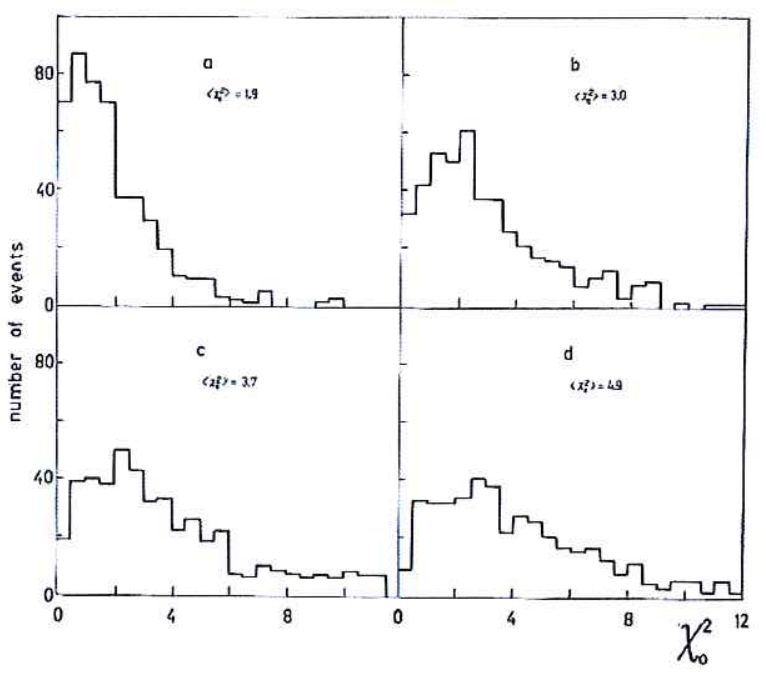

Fig, 7. Distribution of minimized chi-square values in 500 simulated events $\alpha+{ }^{12} \mathrm{C} \rightarrow \alpha+{ }^{6} \mathrm{Li}+{ }^{6} \mathrm{Li}$. 
to the physically correct event (not deformed), $\sigma_{\mathrm{m} n}$ : errors on $x_{\mathrm{m}}$,

$\sigma_{\mathrm{f}}$ : calculated errors on $x_{\mathrm{f}}$ after fit.

In figs. 5 and 6 we represent the distributions $x_{\mathrm{m}}-x_{0} / \sigma_{\mathrm{m}}$ and the "pulls" $x_{\mathrm{m}}-x_{0} /\left(\sigma_{\mathrm{m}}^{2}+\sigma_{\mathrm{f}}^{2}\right)^{\frac{1}{2}}$ for 500 simulated events $\alpha+{ }^{11} \mathrm{C} \rightarrow \alpha+{ }^{6} \mathrm{Li}+{ }^{6} \mathrm{Li}$. Both distri. butions should be Gaussian, centered at zero and with unit standard deviation. The distributions of figs. 5 and 6 show if deformation is coherent with calculated crrors. In fig. 5b and $\mathrm{c}$ are overestimated and underestimated respectively. Distributions of $\chi^{2}$ (fig. 7) and associated probability (fig. 8) are related to those of figs. 5 and 6 . As the number of degrees of freedom for the example chosen is $v=4$, the value of the $\chi^{2}$ is 4 and the $\chi^{2}$ distribution has a maximum at $\chi^{2}=v-2=2$. In fig. 7 it is apparent that the $\chi^{2}$ distribution has the correct shape only when errors have been correctly evaluated. On the other hand, the shape of the probability distribution is uniform when errors are correctly estimated, as is shown in fig. 8. In fig. 9 the $\chi^{2}$ and associated probability distributions are shown for 300 simulated events with a neutral particle. In this case, $y=1$ and the mean yalue of the $\chi^{2}$ is 1 . The maximum of the distribution must be at the otigin.

\section{Application to real experiment}

The method has been applied up to now to the identification of 1000 interactions of $135 \mathrm{MeV}$ protons and over 3000 interactions of $45 \mathrm{MeV} \alpha$-particles with $\mathrm{C}$,

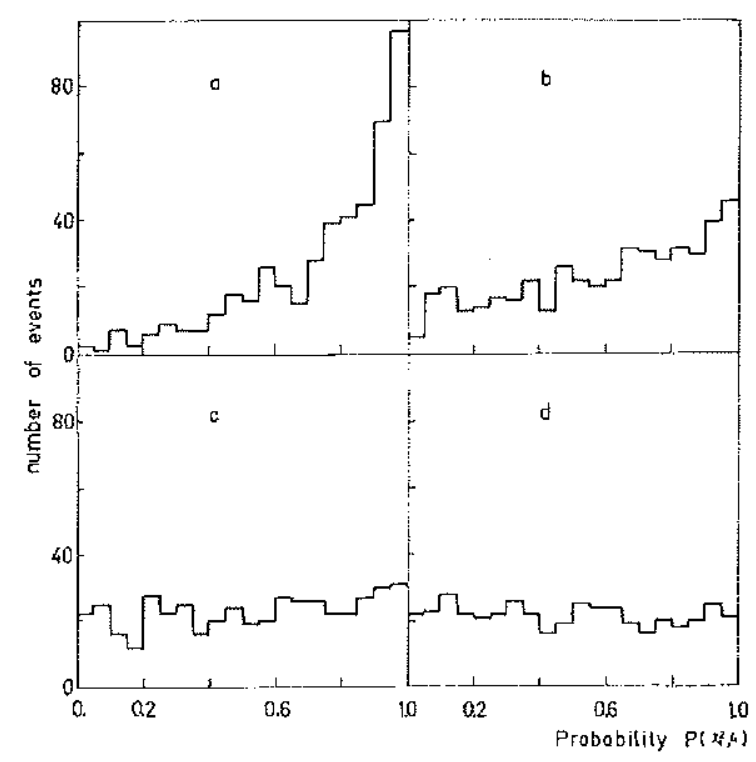

Fig. 8. Distribution of associated probabilities in 500 simulated events $\alpha+{ }^{12} \mathrm{C} \rightarrow \alpha+{ }^{6} \mathrm{Li}+{ }^{6} \mathrm{Li}$.
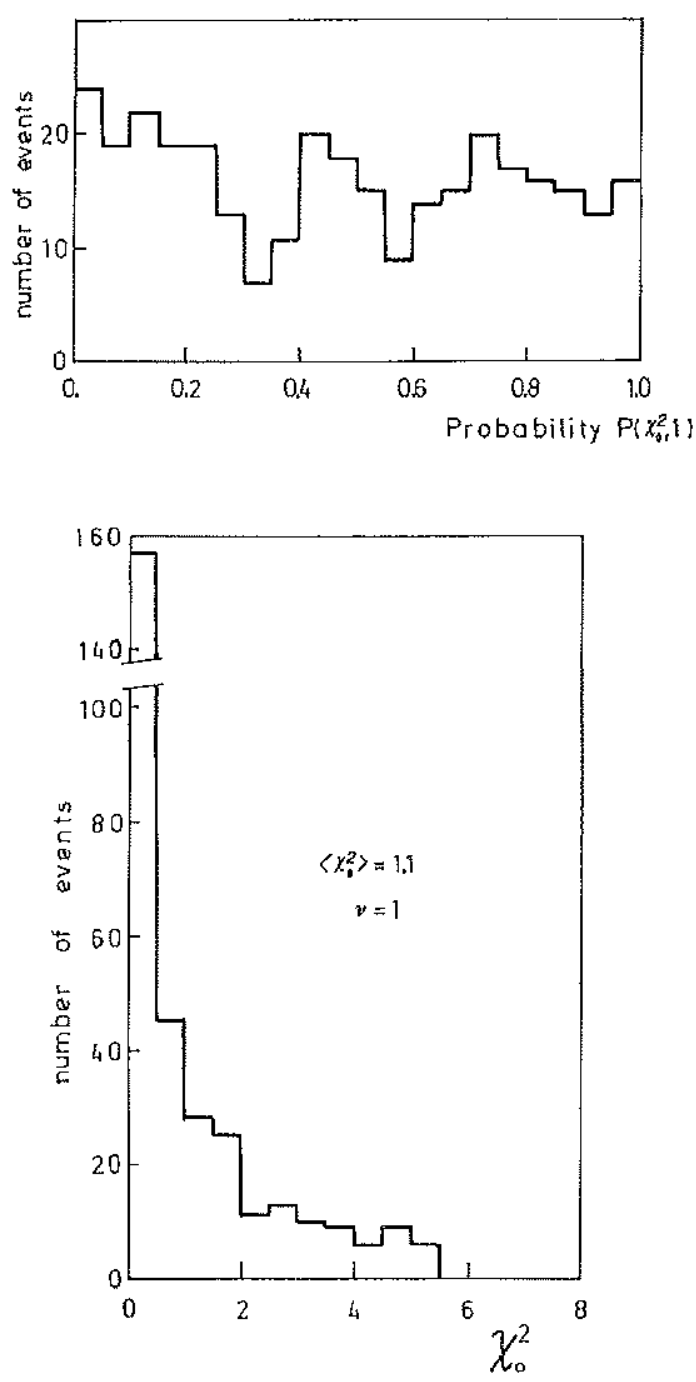

Fig. 9. Distributions of minimized chi-scuare and associated probability in 300 simulated events $\mathrm{p}+{ }^{12} \mathrm{C} \rightarrow \mathrm{n}+{ }^{7} \mathrm{Be}+{ }^{7} \mathrm{Be}$.

$\mathrm{N}$, $\mathrm{O}$ targets in nuclear emulsion and numbers of prongs $N \geqq 4$. Reaction cross sections and production yields of isotopes of astrophysical interest $6 \leqq A \leqq 11$ have been determined ${ }^{12,13}$ ) and will be promptly published.

High statistics obtained on certain channels and the precise knowledge of the kinematics furnished by the identification method will permit investigation on spectroscopy of excited states of light nuclei.

The authors want to express their gratitude to the French Ministry of Foreign Affairs, the Spanish Ministry of Education and Science and the Nuclear 
Energy Comission for valuable support. Colaboration and assistance from the Center of Data Processing of the Ministry of Education and Science in Madrid are also very specially acknowledged.

\section{References}

1) M. Jung, C. Jacquot, C. Baixeras, R. Schmitt, H. Braun and L. Girardin, Phys. Rev. 188 (1969) 1517.

$\left.{ }^{2}\right)$ C. Jacquot, Y. Sakamoto, M. Jung, C. Baixeras, L. Girardin and H. Braun, Nucl. Phys. A148 (1970) 325.

3) C. Jacquot, Y. Sakamoto, M. Jung and L. Girardin, Nucl. Phys. A201 (1973) 247.

4) C. Jacquot, Thesis (Strasbourg, 1972).
5) J. P. Berge, UCRL Memo 86 (1959/60).

6) R, Böck, CERN STS 60-30 (1960).

$\left.{ }^{7}\right)$ M. M. Shapiro, Encyclopedia of physics, vol. 45, Nucl. Instr. $11(1958)$.

$\left.{ }^{8}\right)$ E. V. Benton and R. P. Henke, Nucl. Instr. and Meth. 67 (1969) 87.

9) R. P. Henke and E. V. Benton, USNRDL-TR-67-122 (1967)

10) L. Jauneau and D. Morellet, CERN 64-13 (1964).

1i) A. Hastings, Approximations for digital computers (Princeton University Press, Princeton, N.J., 1955).

12) M. Ortega, Thesis (Universidad Autónoma de Barcelona, 1975).

13) A. VidalmQuadras, Thesis (Universidad Autónoma de Barcelona, 1975). 